\title{
Per trasmettere il progetto
}

\author{
Fabrizio Rossi Prodi
}

Come molti colleghi, negli anni scorsi anche io avevo creduto e promosso le riforme ordinamentali, sì da costruire un percorso formativo più adeguato alle necessità contemporanee della figura dell'architetto. E reso forte dall'illusione di poter gestire un inedito potere, ascoltavo scettico i consigli dei più anziani, che garbatamente mi segnalavano - proprio per la loro analoga e precedente esperienza l'attesa inefficacia di quel genere di azioni di riforma. Se non è per ragioni anagrafiche, cioè per essere entrato ormai a far parte degli anziani, vorrei dire che l'esperienza degli ultimi dieci anni e più di riforme dimostra ampiamente che erano profetiche quelle parole di sfiducia, che mi toccava ascoltare nel momento in cui invece proponevo cambiamenti che mi sembravano epocali (geometrie di percorsi formativi, intensificazione di relazioni interdisciplinari, declaratorie di contenuti ecc.).

Aggiungerei che oggi mi sembra stucchevole e certamente attardata anche la querelle fra il $3+2$ e il ciclo unico, quest'ultimo strenuamente difeso dai compositivi della mia sede - e di altre - come ultima frontiera della qualità. Si tratta invero di due percorsi formativi dai contenuti pressoché identici e sono molto scettico su qualsiasi ipotesi di loro trasformazione, perché il vero problema mi pare che non stia negli ordinamenti. Inoltre per entrambi l'accesso è solo illusoriamente governato, perché i numeri sono storicamente in folle eccedenza rispetto alle capacità di assorbimento del mercato, con sovraccarico per le risorse didattiche e minor capacità di trasmissione disciplinare, esubero di architetti in uscita che aggrava lo spreco nella selezione delle professionalità e nella valorizzazione delle competenze di un sistema di acquisizione del lavoro che, prima di essere in crisi, è complessivamente sbagliato.

Mi sembra dunque che, indipendentemente dall'ingegneria ordinamentale e dopo mesi e mesi persi nel vano sforzo di migliorare gli statuti dei singoli corsi, l'unica vera qualità che siamo stati in grado di infondere negli studenti, l'unica risorsa cui davvero potranno ricorrere deriva da un attento e rigoroso esercizio del progetto da parte di noi docenti, dalla sua onesta trasmissione ai giovani allievi e dalla metodica di laboratorio. L'esercizio del progetto da parte dei docenti - e la sua elaborazione teorica e trasmissione didattica - mi sembra il cuore del problema, in un quadro in cui il progetto per vari motivi è poco praticato e i ruoli accademici si vanno riempiendo non già di architetti, ma di letterati di architettura.

Quando i nuovi laureati si confrontano con la rarefazione delle occasioni professionali, col moltiplicarsi dei saperi tecnici, quando saranno disorientati per i nuovi strumenti di disegno e di visualizzazione, quando saranno stretti nei risparmi di budget o nell'asfissia burocratica, probabilmente posti nella necessità di emigrare e confrontarsi con culture molto diverse, a fronte di tutto questo, l'unico solido presidio disciplinare su cui potranno fondare la propria azione rimarrà non tanto gli specialismi, ma la competenza nell'esercizio del progetto - se saremo stati in grado di trasmetterla a loro - cioè quella capacità di elaborare un pensiero e tradurlo in forme e spazi, di mettere insieme cose diverse, dialogando con i luoghi, con altri sistemi, con le diverse tecniche, ma sempre guidati da un processo consapevole e un'interpretazione umanistica che fa 


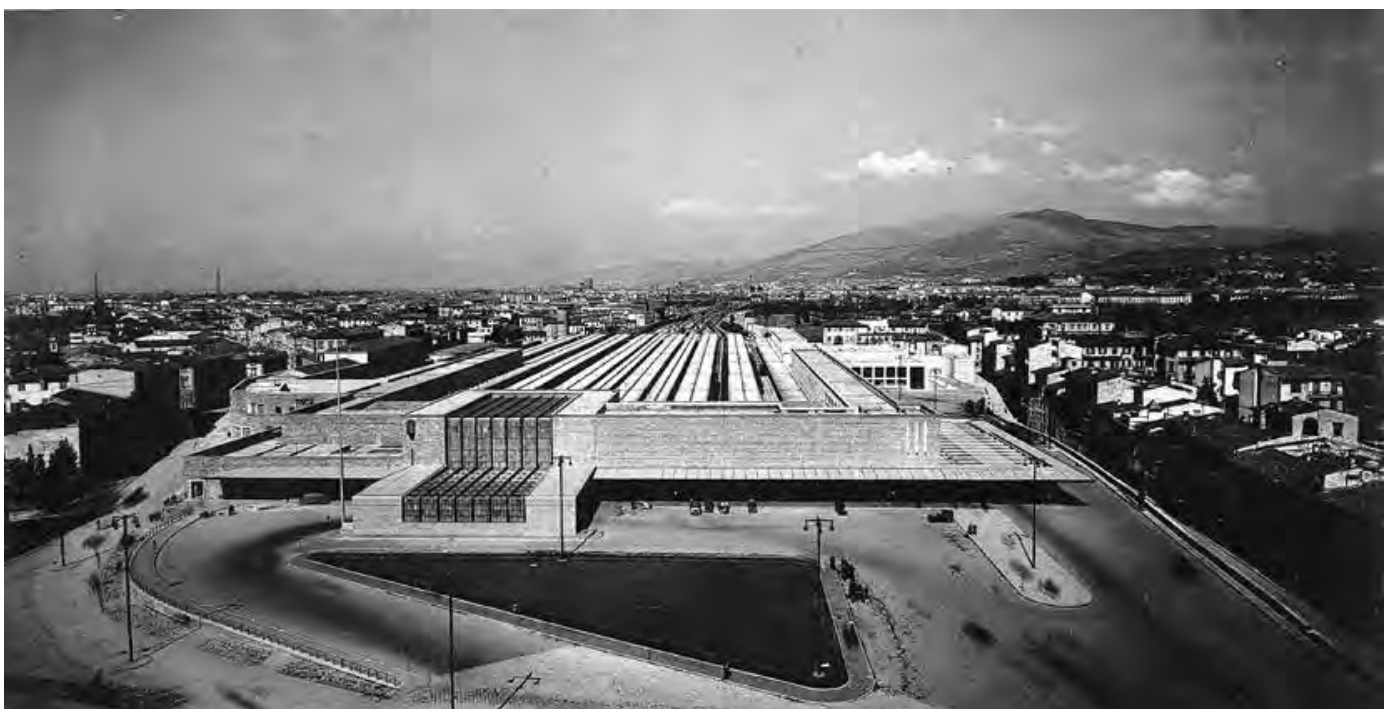

I. Pier Niccolò Berardi, Nello Baroni, Italo Gamberini, Sarre Guarnieri, Leonardo Lusanna, Giovanni Michelucci, stazione Santa Maria Novella. RRD-Raccolta Roberto Dulio.

salva la finalità sociale e un principio etico di responsabilità nei confronti delle trasformazioni dell'ambiente.

Poiché guardo con interesse alle innovazioni e cerco di introdurle nei progetti che sviluppo, penso di poter dire con ragionevole certezza che non vedo proiezione nel futuro $\mathrm{o}$ innovazione digitale, o novità tecniche, o ancora esotismo di luoghi che possano scalfire questo nucleo resistente della formazione o quantomeno che non possano essere riassorbiti negli strumenti e nei metodi appresi per l'esercizio del progetto di architettura e di paesaggio. Stiamo affrontando gli algoritmi e i big data, siamo alle soglie dell'intelligenza artificiale, vediamo scenari più ampi e complicati, con metodi che cambiano e paradigmi che si succedono, ma i fattori di più di lunga durata del progetto permangono come invarianti resistenti: è l'unica specificità che ci sostiene e che, secondo me, è destinata a durare, l'unico vero contenuto che dobbiamo cercare di trasmettere.

Naturalmente, la certezza di disporre di un metodo non significa rimanere fissi con lo sguardo à rebour, o illudersi di poter sfuggire al confronto con la modernità, in particolare in Italia dove invece tutta la storia non solo culturale, ma anche civile del Paese è segnata da questo sforzo di importare (talvolta acriticamente) proprio la modernita dal Nord $\mathrm{o}$ dall'Occidente. Tutt'altro, sono convinto che nei nostri insegnamenti e nella ricerca - quin- di anche nella pratica del progetto - debbano trovare luogo le nuove istanze sociali e multietniche, il nuovo modo di intendere lo spazio pubblico, gli spazi intermedi, i nuovi sistemi di visione virtuale, tutto il digitale, la gestione di dati in crescita esponenziale, l'interdisciplinarietà, il modo in cui la comunicazione cambia il progetto, insomma tutte le innovazioni di contenuto e di metodo che stanno accompagnando questi ultimi due decenni. E francamente non trovo resistenze, anzi riscontro un vivissimo interesse sia in me, che negli allievi, a coltivare la sperimentazione per aggiornare - marginalmente - le tecniche più tradizionali di progetto.

Alcuni di questi elementi di forte innovazione già rientrano nei programmi di insegnamento dei nostri corsi di studio, ma in prevalenza si tratta di temi o specialismi appropriati alla ricerca sperimentale o ai corsi di terzo livello; sono temi importanti, ma non tali da ridurre il tempo che invece deve essere destinato alle questioni di base per la formazione generalista dell'architetto, umanistica e tecnica, teorica e pratica, questa sì, indispensabile. Perché sovente accade, e soprattutto in Italia, di essere affetti da presbiopia: ci preoccupiamo moltissimo di mettere a fuoco visioni lontane per ritrovare una direzione di marcia, e non ci accorgiamo di avere, proprio vicino a noi, le migliore risorse per muoverci.

Proprio per delineare con chiarezza i temi e le specificità didattiche e di ricerca della 
mia Scuola, quindici anni fa avevo tentato un bilancio del suo pensiero compositivo, con un libro Carattere dell'architettura toscana, che vedo risulta attuale ancora oggi. Il testo si interrogava sull'esistenza di un carattere specifico e originale espresso dall'architettura toscana e dalla sua Scuola nel corso del Novecento, capace di tenere insieme i lineamenti di più lunga durata con le trasformazioni indotte dal Moderno, e se esso era specifico e diverso da quello di altre realtà regionali. In sostanza mi chiedevo se esiste una particolare sensibilità per alcuni aspetti compositivi o per alcuni strumenti interpretativi, in modo da delineare il filo del pensiero teorico sulla composizione architettonica, almeno dagli anni Trenta, che poi si traduce in esercizio del progetto e in trasmissione didattica ai fini della formazione, creando alla fine una circolarità che si deposita nelle forme del paesaggio toscano. Gli esiti erano stati interessanti, perché riscontravano un legame fra alcuni caratteri secolari dell'architettura e del paesaggio di questa regione, con certi metodi e caratteri dei Maestri del Novecento e con la loro trasmissione disciplinare nella Scuola. Vedo oggi che la loro azione permane ancora ai nostri giorni e delinea in fondo il patrimonio genetico della Scuola, la ritrovo ancora nei progetti e nella didattica della generazione cui appartengo. Avevo raggruppato in cinque fattori i nuclei resistenti di questo patrimonio, che ancora oggi trovo valido e degno di essere coltivato e trasmesso.

Al primo punto riscontravo l'abitudine costante a ricercare una fondazione etica nel progetto, di solito ritrovata nella dimensione comunitaria dello spazio architettonico e in origine legata alla matrice religiosa dell'architettura toscana, oltre che al fattore di coesione e trasmissione svolto dalla bottega artigiana in antichità e dagli spazi di lavoro della fabbrica più recentemente. È il riconoscimento della centralità dell'uomo nel progetto e la non meno importante conseguenza che un principio di razionalità guida sempre e ordina il progetto, visto non come bel disegno, ma come processo di pensiero, lavoro di pensiero su apparati tematici, dunque un pensiero che lavora sul pensiero di altri e, così, attua una trasformazione. A questa centralità umanisti$\mathrm{ca}$ - che ha un'ascendenza rinascimentale, non meno che illuminista - e alla razionalità logi-

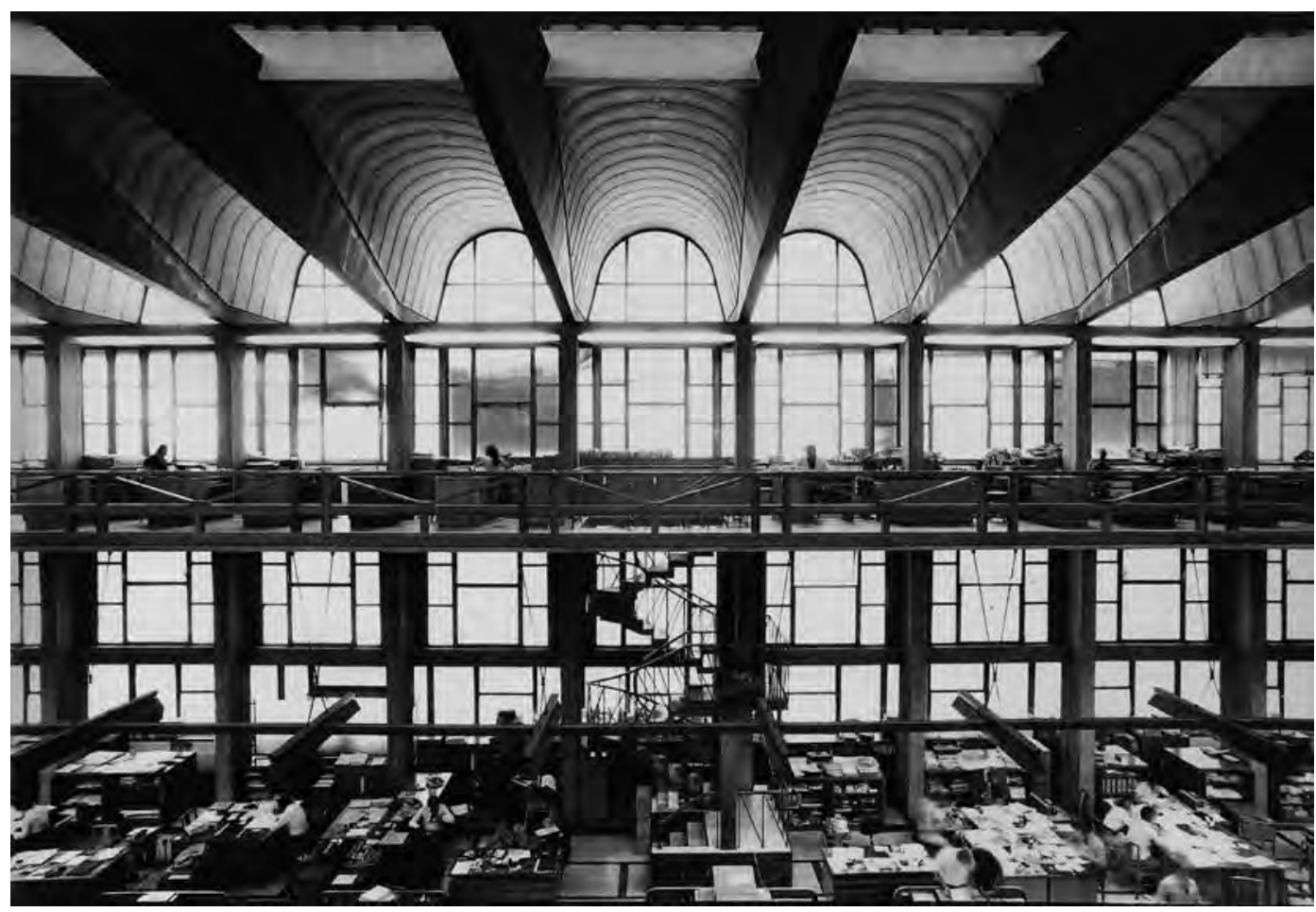

2. Giovanni Michelucci, salone della Cassa di Risparmio di Firenze, 1957. Archivio Fondazione Michelucci. Foto Arrigo Coppitz. 


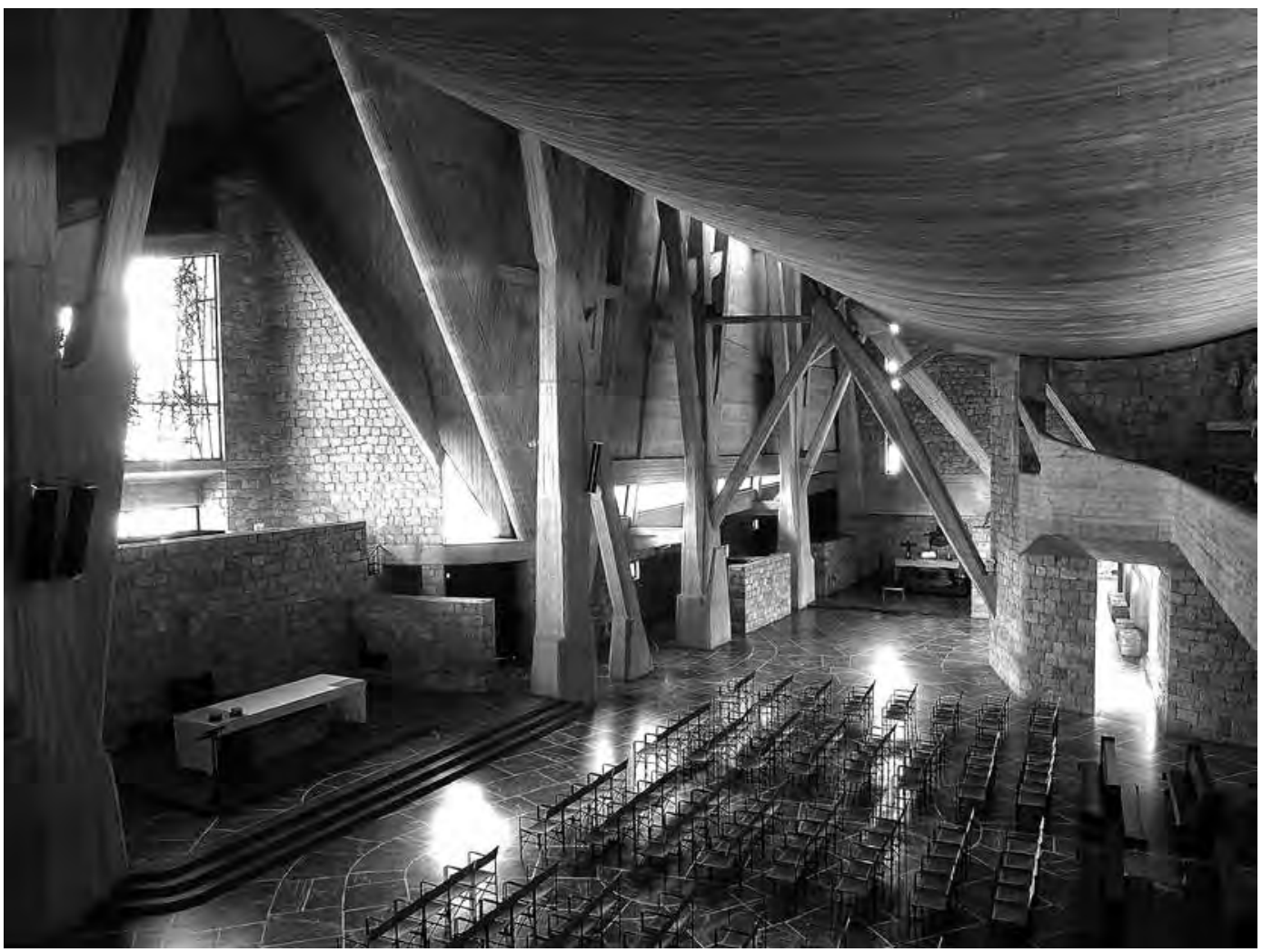

3. Giovanni Michelucci, chiesa dell’Autostrada del Sole, Campi Bisenzio, I964. Archivio Fondazione Michelucci. Foto Aleardo Aleardi, 20 I I.

ca delle scelte progettuali, viene riconosciuto il presupposto morale del mestiere. In questi due fattori - umanesimo e razionalità - si può identificare il motore dell'invenzione spaziale, l'impulso per un grande rinnovamento figurativo e sintattico, talvolta anche eccessivo, perché spesso trasceso in un criticismo eccessivo dell'apparato figurativo, compositivo e metodologico, come il dubbio metodico michelucciano ci mostra, allorquando è sembrato cadere nella sistematica distruzione dei codici e addirittura nell'iconoclastia.

Il secondo punto si occupava della nascita dell'approccio linguistico, riconoscendone la genesi in quel criticismo già ricordato, tipico della nostra area culturale e che, nel campo specifico dell'architettura, aveva favorito un'attitudine a disarticolare, a scomporre e ad assemblare gli elementi, misurandoli in relazione alla percezione umana. Questo approccio si riscontra nelle poetiche di singoli autori, ma fu anche la base per una nuova metodica di lettura compositiva delle opere, inquadrando in modo inedito per quegli anni e anticipatore della grande stagione ermeneutica che dopo è cominciata e da poco è finita - l'architettura come un linguaggio costituito da segni, articolato in valori grammaticali e sintattici, poi raggruppati in classi e variamente legati alle funzioni e all'interpretazione fenomenologica degli utenti. È l'innovazione introdotta nella teoria compositiva da Gamberini e Koenig, anche con l'aiuto di Eco, passando dalle disarticolazioni fisiche, alle funzioni, fino alle forme significanti. Queste figure vengono prevalentemente ricercate e tratte dal paesaggio toscano e dall'architettura minore, dalle relazioni fra le parti e da un'interpretazione corale dei fatti urbani. Su questa linea ritroviamo più tardi Natalini che codifica le figure di pietra, la memoria e gli archetipi, ritrovo buona parte della mia ricerca e anche quella di Paolo Zermani, ritrovo le ricerche di Dottorato della nostra sede, in gran parte impegnate sui temi dei linguaggi, della resistenza alle trasformazioni indotte dai nuovi mezzi di comunicazione, della ricerca dell'identità e del senso di lunga durata dei luoghi e delle istituzioni. 
Il terzo punto riguarda il rapporto genetico fra città e architettura, in genere mai visto come approccio generalizzante e strutturale sulle logiche urbane, ma più come canone impressionistico, come testimonia la definizione michelucciana di «effetto città» e ancora di «città variabile». $\grave{E}$ prevalentemente la stagione medievale che costituisce il modello da cui sono tratte le figure trascritte nei progetti e le loro regole di aggregazione, con una riduttiva interpretazione delle questioni urbane, semmai, viste più come sistemi di relazione e fatti linguistici, che non come occasioni per inquadrare la ragion d'essere della città, la sua struttura: un approccio sorprendentemente congruente con gli scenari delineati dal dibattito architettonico contemporaneo. In anni recenti ho anche ritrovato in queste posizioni un'eco dei limiti brunelleschiani a inquadrare il rapporto fra architettura e città, quando il Maestro moltiplica i suoi moduli prospettici nello sforzo di ritrovare questo rapporto, ma manca di agguantare la ragion d'essere della città e la sua riforma, arrivando tutt'al più a costruire delle architetture urbane, ma pur sempre della architetture, giusto a una scala un po' maggiore.

Un quarto gruppo di argomenti riguarda alcune inclinazioni progettuali più tipiche $\mathrm{e}$ ricorrenti nei protagonisti della Scuola, spesso presentate come opposizioni o endiadi: tra queste la dialettica tra principio d'ordine e principio di disarticolazione, il rapporto fra geometria e costruttività, con la costruttività che consuma la purezza geometrica delle forme, la murarietà severa opposta alla spazialità interna, lo studio in sezione per immaginare lo spazio interno e il suo omologo in pianta con il «piano di vita», l'anatema nei confronti della facciata, le espressioni volumetriche, gli spigoli e le ombre ecc. Sono tutti tratti che delineano con costanza il patrimonio culturale e il bagaglio disciplinare di lunga durata della Scuola.

Concludevo quel mio testo con un capitolo sulla tradizione e sul suo ruolo nella Scuola, in cui registravo ancora una volta una predilezione per le espressioni corali e minori, a scapito della tradizione maggiore, celebrata dai libri, un interesse per il paesaggio naturale e urbano e per l'architettura rurale. Vi riconoscevo uno sforzo di radicamento linguistico nei luoghi e nella storia di lunga durata, che induce a comporre il progetto con i materiali trovati nei luoghi, in una strategia di trascrizione o di ri-scrittura, in cui il disegno è strumento di indagine compositiva, di misurazione dei luoghi e di conservazione delle identità, proprio risorse e presidi per affrontare gli scenari di cambiamento sollecitati dall'epoca contemporanea.

Gran parte di questi temi e di queste consuetudini compositive viene ancora oggi approfondita, dibattuta e posta a confronto con i recenti cambi di paradigma o con le nuove tecniche, sia nei convegni dell'Identità dell'Architettura Italiana, che nella rivista «Firenze Architettura», o nelle ricerche e pubblicazioni, ma soprattutto nell'esercizio del progetto, e segnatamente nella ricerca di concorso, cui molti docenti della Scuola con costanza si dedicano come occasione sperimentale di verifica e come ricerca operativa sui contenuti e sui metodi.

Proprio questi lineamenti costituiscono in definitiva il patrimonio genetico della Scuola e si ritrovano in misura diversa attraverso complicate trame genealogiche nei suoi docenti ma anche nei paesaggi circostanti, fino a oggi senza soluzione di continuità. Si tratta di regole e anche di figure compositive che appartengono a un codice condiviso, costruito in secoli e affinato negli ultimi decenni, sono l'espressione di una comunità che ancora le usa, le trasforma, ne misura la tenuta e le sperimenta. Credo che si tratti ancora del nucleo di strumenti che abbiamo custodito, coltivato e che possiamo affidare alle generazioni più giovani, perché guardino con fiducia al futuro. 